\title{
Differential effects of operative complications on survival after surgery for primary lung cancer
}

\author{
Felix G. Fernandez, MD, MSc, ${ }^{a}$ Andrzej S. Kosinski, PhD, ${ }^{\mathrm{b}}$ Anthony P. Furnary, MD, ${ }^{\mathrm{d}}$ Mark Onaitis, MD,

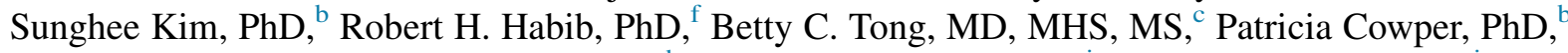 \\ Daniel Boffa, MD, ${ }^{g}$ Jeffrey P. Jacobs, MD, ${ }^{\mathrm{h}}$ Cameron D. Wright, MD, ${ }^{\mathrm{i}}$ and Joe B. Putnam, MD
}

\section{ABSTRACT}

Objective: Complications adversely affect survival after lung cancer surgery. We tested the hypothesis that effects of complications after lung cancer surgery on survival vary substantially across the spectrum of postoperative complications.

Methods: The Society of Thoracic Surgeons General Thoracic Surgery Database was linked to Medicare data for lung cancer resections from 2002 through 2013. Linkage was achieved for 29,899 patients. A survival model was created that included operative complications as explanatory variables and adjusted for relevant baseline covariates. Because of violation of the proportional hazard assumption, we used time-varying coefficient Cox modeling for the complication variables.

Results: Median patient age was 73 years, and 48\% were male. Procedures performed were lobectomy in $69 \%$, wedge in $17 \%$, segmentectomy in $7 \%$, bilobectomy in 3\%, pneumonectomy in 3\%, and sleeve lobectomy in $1 \%$. Most frequent complications were atrial arrhythmia (14\%), pneumonia $(4.3 \%)$, reintubation $(3.8 \%)$, delirium $(2 \%)$, and acute kidney injury $(1.4 \%)$. In the early period (090 days), 12 complications are associated with worse survival. From 3 to 18 months after surgery, only 4 complications are associated with survival: delirium, blood transfusion, reintubation, and pneumonia. After 18 months, only sepsis and blood transfusion are associated with a significant late hazard.

Conclusions: Our analysis confirmed the presence of differential magnitude and time-varying effects on survival of individual complications after lung cancer surgery. We conclude that the derived time-dependent hazard ratios can serve as objective weights in future models that enhance performance measurement and focus attention on prevention and management of complications with greatest effects. (J Thorac Cardiovasc Surg 2018;155:1254-64)

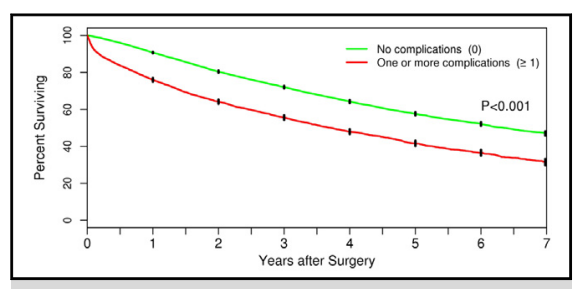

Survivals of patients with and without complications after lung cancer surgery.

\section{Central Message}

A number of perioperative complications after lung cancer surgery are associated with timevarying effects on early, intermediate, and late-term survivals of differential magnitude.

\section{Perspective}

Current models used for risk prediction and performance measurement in lung cancer surgery assign equal weights to empirically selected major morbidities. Complications after lung cancer surgery are associated with different magnitudes of hazard for mortality. These differential hazards can be used as objective weights for complications in risk prediction and performance measurement models.

See Editorial Commentary page 1265.

See Editorial page 1209.
Perioperative complications adversely affect survival after lung cancer surgery. ${ }^{1}$ The Society of Thoracic Surgeons (STS) General Thoracic Surgery Database (GTSD) has developed risk prediction and performance models for

From ${ }^{\mathrm{a} D e p a r t m e n t}$ of Surgery, Emory University School of Medicine, Atlanta, Ga; Departments of ${ }^{b}$ Biostatistics and ${ }^{\mathrm{c}}$ Surgery, Duke University, Durham, NC; ${ }^{\mathrm{d}}$ StarrWood Cardiothoracic Group, Portland, Ore; ${ }^{\mathrm{e} D e p a r t m e n t ~ o f ~ S u r g e r y, ~ U n i v e r s i t y ~ o f ~}$

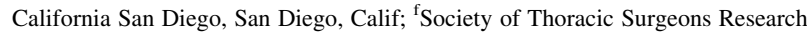
Center, Chicago, Ill; ' ${ }^{\mathrm{g}}$ Department of Surgery, Yale University, New Haven, Conn; ' Department of Surgery, Johns Hopkins All Children's Hospital and Johns Hopkins University, St Petersburg, Fla; ${ }^{\text {i } D e p a r t m e n t ~ o f ~ S u r g e r y, ~ M a s s a c h u s e t t s ~}$ General Hospital, Boston, Mass; and ${ }^{\mathrm{j} B a p t i s t}$ MD Anderson Cancer Center, Jacksonville, Fla.

Supported by grant number R01 HS022279 from the Agency for Healthcare Research and Quality. The content is solely the responsibility of the authors and does not necessarily represent the official views of the Agency for Healthcare Research and Quality. lung cancer surgery that are based on preoperative factors. $^{2,3}$ These models consider operative morbidity and mortality as outcome measures and empirically assign equal weights to major complications. Major morbidities

Read at the 97th Annual Meeting of The American Association for Thoracic Surgery, Boston, Massachusetts, April 29-May 3, 2017.

Institutional review board approval: Pro00055346, approved June 16, 2014.

Received for publication April 13, 2017; revisions received Aug 30, 2017; accepted

for publication Sept 9, 2017; available ahead of print Dec 6, 2017.

Address for reprints: Felix G. Fernandez, MD, MSc, The Emory Clinic, 1365 Clifton

Rd NE, Suite A2214, Atlanta, GA 30322 (E-mail: felix.fernandez@ emoryhealthcare.org).

$0022-5223 / \$ 36.00$

Copyright (C) 2017 by The American Association for Thoracic Surgery

https://doi.org/10.1016/j.jtcvs.2017.09.149 


\section{Abbreviations and Acronyms \\ ARDS = adult respiratory distress syndrome \\ CMS $=$ Centers for Medicare and Medicaid Services \\ GTSD $=$ General Thoracic Surgery Database \\ ICD-9-CM = International Classification of \\ Diseases, Ninth Revision, Clinical \\ Modification \\ STS $=$ Society of Thoracic Surgeons}

Scanning this $\mathrm{QR}$ code will take you to a supplemental table. To view the AATS Annual Meeting Webcast, see the URL next to the webcast thumbnail.

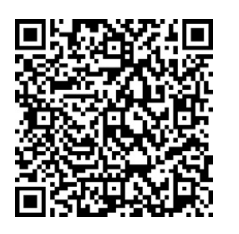

were selected by expert opinion and include tracheostomy, reintubation, initial ventilatory support longer than 48 hours, adult respiratory distress syndrome (ARDS), bronchopleural fistula, pulmonary embolus, pneumonia, unexpected return to the operating room, and myocardial infarction. Other complications, such as an atrial arrhythmia requiring treatment, are considered minor and not included in these models.

Not all postoperative complications, however, would be expected to be associated with the same risk of mortality. The assignment of equal weights to complications in risk and performance measurement models thus may not be appropriate. In addition, the death hazard from a specific complication may not be constant with time. An acute pulmonary embolus may be associated with a significant risk in the immediate perioperative period but have no effect on survival at 2 years after the operation. Our objective was to determine the independent effects of perioperative complications of lung cancer surgery on survival with time. Long-term survival for lung cancer surgeries in the STS GTSD was ascertained through linkage with Medicare claims data. ${ }^{4}$ We tested the hypothesis that the effects of complications after lung cancer surgery on survival vary substantially across the spectrum of postoperative complications.

\section{MATERIALS AND METHODS \\ Institutional Review Board Approval}

The data used in this analysis represent a limited data set with no direct patient identifiers that was originally collected for nonresearch purposes. The analysis of these data was therefore declared by the Duke University Health System Institutional Review Board to be research not involving human subjects and thus determined to be exempt from formal review.

\section{Society of Thoracic Surgeons General Thoracic Surgery Database}

The STS GTSD was queried for all patients treated with surgical resection for primary lung cancer from January 1, 2002 through December 31, 2013. This study encompassed several versions of the STS GTSD data collection instruments (v2.07, v2.081, and v2.2), with various changes incorporated to reflect advancement in thoracic operations.

\section{Medicare Database of CMS}

Medicare is health insurance provided by the federal government of the United States for the following groups of patients: (1) people aged 65 years or older, (2) people younger than 65 with certain disabilities, and (3) people of any age with end-stage renal disease. The Centers for Medicare and Medicaid Services (CMS) administrative claims data source for this study is the $100 \%$ Medicare inpatient claims file, which contains information on hospitalizations of patients enrolled in fee-for-service Medicare. It includes dates of service and diagnostic codes from the International Classification of Diseases, Ninth Revision, Clinical Modification (ICD-9-CM). The database contains anonymous patient identifiers that enable follow-up of beneficiaries with time but do not enable identification of any beneficiary through assigned Medicare health insurance number. Also used was the $100 \%$ Medicare denominator file, which links to the inpatient file and contains information on beneficiary eligibility, demographic characteristics, and date of death.

\section{Linkage of STS Records to CMS Data}

The STS database was linked to CMS claims files with combinations of nonunique indirect identifiers through a deterministic matching algorithm, as previously described. ${ }^{4}$ Records in the 2 databases were considered to contain information on the same patient if they matched on a set of indirect identifiers, including hospital, date of birth, sex, admission date, and discharge date. The CMS $100 \%$ inpatient claims file was first queried for ICD-9-CM procedure codes for pulmonary resections 32.0 to 32.9. Variables common to both the STS GTSD and the CMS Medicare Database were then used to link STS operations to CMS inpatient claims data for all hospitalizations during which a patient underwent lung cancer resection. Once the individual patients were linked, longitudinal records were created containing follow-up information, including subsequent death where applicable. Successful linkage was performed for 29,899 patients who underwent lung cancer resection.

\section{Outcome Measure and Explanatory Variables}

The objective of this study was to examine the differential effects of operative complications after lung cancer surgery and overall survival; the primary outcome measure is therefore mortality. Date of death was determined from the CMS database for each patient, whereas censorship was based on the last year of CMS follow-up that was available. Operative complications occurring in the immediate inpatient setting captured in the STS-GTSD that were considered as explanatory variables include the following: pneumonia, pulmonary embolus, bronchopleural fistula, ARDS, reintubation, initial ventilation for longer than 48 hours, atrial arrhythmia requiring treatment, ventricular arrhythmia requiring treatment, myocardial infarction, deep vein thrombosis, empyema, surgical site infection, sepsis, recurrent laryngeal nerve paralysis, central neurologic event, delirium, acute kidney injury, blood transfusion, and unexpected return to the operating room (for any cause). These complications are typically ascertained by data abstractors through review of the medical record. The following independent variables were also adjusted for: age, sex, body mass index, American Society of Anesthesiology Risk class, Zubrod score, coronary artery disease, cerebrovascular disease, congestive heart failure, diabetes mellitus, steroid use, peripheral vascular disease, renal 
TABLE 1. Patient characteristics

\begin{tabular}{|c|c|c|}
\hline Characteristic & No. & $\% *$ \\
\hline \multicolumn{3}{|l|}{ Age (y) } \\
\hline $65-69$ & 8671 & 29.0 \\
\hline $70-74$ & 8886 & 29.7 \\
\hline $75-79$ & 7147 & 23.9 \\
\hline$\geq 80$ & 5195 & 17.4 \\
\hline \multicolumn{3}{|l|}{ Sex } \\
\hline Male & 14,412 & 48.2 \\
\hline Female & 15,474 & 51.8 \\
\hline \multicolumn{3}{|l|}{ BMI $\left(\mathrm{kg} / \mathrm{m}^{2}\right)$} \\
\hline$<18.5$ & 794 & 2.7 \\
\hline $18.5-25$ & 9568 & 32.0 \\
\hline $25-30$ & 12,323 & 41.2 \\
\hline $30-35$ & 4961 & 16.6 \\
\hline$>35$ & 2253 & 7.5 \\
\hline \multicolumn{3}{|l|}{ ASA class } \\
\hline I-II & 3885 & 13.1 \\
\hline III & 22,281 & 75.4 \\
\hline IV-V & 3391 & 11.5 \\
\hline \multicolumn{3}{|l|}{ Zubrod score } \\
\hline 0 & 12,443 & 42.0 \\
\hline 1 & 15,451 & 52.2 \\
\hline $2-5$ & 1700 & 5.7 \\
\hline CAD & 7959 & 26.6 \\
\hline CVD & 3000 & 10.0 \\
\hline CHF & 1152 & 3.9 \\
\hline DM & 5677 & 19.0 \\
\hline Steroid use & 1036 & 3.5 \\
\hline PVD & 3599 & 12.0 \\
\hline Renal insufficiency & 697 & 2.3 \\
\hline \multicolumn{3}{|l|}{$\mathrm{FEV}_{1}(\%$ predicted $)$} \\
\hline$>80$ & 13,613 & 50.4 \\
\hline $60-80$ & 8637 & 32.0 \\
\hline $40-60$ & 3910 & 14.5 \\
\hline$<40$ & 842 & 3.1 \\
\hline \multicolumn{3}{|l|}{ Pathologic stage } \\
\hline I & 20,038 & 68.9 \\
\hline II & 5155 & 17.7 \\
\hline III & 3311 & 11.4 \\
\hline IV & 590 & 2.0 \\
\hline \multicolumn{3}{|l|}{ Cigarette use } \\
\hline Never & 3819 & 12.8 \\
\hline Past & 20,645 & 69.0 \\
\hline Current & 5435 & 18.2 \\
\hline Thoracic reoperation & 1624 & 5.7 \\
\hline \multicolumn{3}{|l|}{ Procedure } \\
\hline Lobectomy & 20,578 & 68.8 \\
\hline Bilobectomy & 842 & 2.8 \\
\hline Pneumonectomy & 915 & 3.1 \\
\hline Segmentectomy & 2079 & 7.0 \\
\hline Sleeve lobectomy & 282 & 0.9 \\
\hline Wedge resection & 5203 & 17.4 \\
\hline VATS approach & 14,182 & 47.4 \\
\hline
\end{tabular}

$B M I$, Body mass index; $A S A$, American Society of Anesthesiologists; $C A D$, coronary artery disease; $C V D$, cardiovascular disease; $C H F$, congestive heart failure; $D M$, diabetes mellitus; $P V D$, peripheral vascular disease; $F E V_{l}$, forced expiratory volume in 1 second; VATS, video-assisted thoracic surgery. *Percentage of all patients. insufficiency, forced expiratory volume in 1 second (\% predicted), smoking status (current, former, never), laterality, whether the patient had a previous thoracic surgery that affected the operative field (ie, thoracic reoperation), procedure type, and whether video-assisted thoracic surgery was used. Lung cancer pathologic stage was also considered, as defined according to the American Joint Committee on Cancer seventh edition staging system. Stage assignment was based on available T, N, and $\mathrm{M}$ descriptors recorded in the STS GTSD. ${ }^{4}$ The STS GTSD has been externally audited since $2010 .^{5}$ Audits have demonstrated high agreement rates with hospital records and validated the accuracy and completeness of the data. All variables, including the operative complications, were selected empirically on the basis of STS operative risk models and expert consensus. Overall, the rate of missing data was low (average of 3\% across the data fields studied). The greatest number of instances of missing data was for the variable of forced expiratory volume in 1 second (missing in around $10 \%$ of patients). For a number of variables (comorbidities and whether the surgery represented a reoperation), failure to code the presence of a variable was considered to be a negative response. The rate of no response ranged from $0.2 \%$ (smoking status) to $7.6 \%$ (perioperative steroids). Model refinement for the Cox proportional hazards model was limited to cases that were complete for all data points considered (those with implied negative responses were considered complete).

\section{Statistical Analysis}

Descriptive analyses are presented as counts with percentages. The Kaplan-Meier method was used to estimate survival after lung cancer surgery in the STS-CMS linked cohort for strata of patients with and without a specific postoperative complication (eg, pneumonia), and the survival curves were compared with the log-rank test. Cox proportional hazards modeling was used to create a long-term multivariable survival model, examining the effects of individual complications on survival with adjustment for the aforementioned patient and operative factors (independent variables). Proportionality of hazards for a complication was evaluated by testing for a correlation of follow-up time with scaled Schoenfeld residuals from the adjusted Cox model with this complication. ${ }^{5}$ Violation of the proportional hazards assumption was observed for most of the operative complication covariates. For each complication, a time-dependent coefficient analysis was therefore considered. To provide a parsimonious analysis but also to allow the estimation of a differential effect of a complication with time, the complication hazard ratios were estimated separately in 3 time intervals: early ( $0-90$ days from operation), intermediate term (3-18 months), and late term (>18 months). The 18-month threshold was based on a visual inspection of time-dependent coefficient plots for complications. ${ }^{6}$ The 90-day threshold was selected empirically to distinguish between the effects of complications on short term survival (operative mortality) and their effects on longer term survival. Clustering within hospitals was accounted for by consideration of robust sandwich covariance matrix estimates. $^{7}$ Analyses were run with the SAS Software 9.4 (SAS Institute, Cary, NC) and R software 3.1.2. ${ }^{8}$

\section{RESULTS}

A query of the STS GTSD from January 1, 2002, through December 31, 2013, revealed 29,899 patients from 247 centers who had undergone surgery for primary lung cancer. Baseline patient characteristics are depicted in Table 1. Median age was 73 years (interquartile range, 69-78 years), and $48 \%$ of the patients were male. At pathologic analysis, $69 \%$ had stage I disease, $18 \%$ had stage II disease, $11 \%$ had stage III disease, and $\%$ had stage IV disease. Procedures performed were lobectomy in $69 \%$, bilobectomy in $3 \%$, pneumonectomy in $3 \%$, segmentectomy in $7 \%$, sleeve 
lobectomy in $1 \%$, and wedge resection in $17 \%$. A thoracoscopic approach was performed in $47 \%$ of operations.

The rates of individual complications after lung cancer surgery are shown in Table 2 . Overall, $27.2 \%$ of patients $(\mathrm{n}=8145)$ had a postoperative complication. The most frequent complications were atrial arrhythmia at $14 \%$ $(\mathrm{n}=4166)$, blood transfusion at $8.5 \%(\mathrm{n}=2527)$, pneumonia at $4.3 \%(\mathrm{n}=1285)$, reintubation at $3.8 \%$ $(\mathrm{n}=1134)$, return to the operating room at $2.9 \%$ $(\mathrm{n}=862)$, delirium at $2 \%(\mathrm{n}=597)$, and acute kidney injury at $1.4 \%(\mathrm{n}=417)$. The operative mortality (30 day or in-hospital mortality) was $2.4 \%(\mathrm{n}=28)$, and the 90 day mortality was $4.5 \%(\mathrm{n}=1343)$. Overall, patients who had 1 or more complications exhibited substantially worse unadjusted survival (Figure 1). Unadjusted survival curves showed that for each specific complication, patients with that complication had worse survival than those without. Figures 2 and 3 demonstrate survival in patients with and without pneumonia, reintubation, ARDS, and blood transfusion.

The differential effects of these complications with time are alternatively depicted in a forest plot in Figure 4 and listed in Table 3. These data demonstrate the adjusted time-dependent hazard ratios for each complication after lung cancer surgery. In the early period of 0 to 90 days (3 months), 12 of 19 complications were associated with worse survival, including pneumonia, pulmonary embolus, bronchopleural fistula, ARDS, reintubation, atrial arrhythmia requiring treatment, ventricular arrhythmia requiring treatment, myocardial infarction, empyema, sepsis, central neurologic event, acute kidney injury, and blood transfusion. Delirium, blood transfusion, reintubation, and pneumonia were associated with increased intermediate-term death hazard. Only sepsis and perioperative blood transfusion continued to influence survival in the late term or after 18 months. Finally, to assess the generalizability of this data to all patients in the STS GTSD, we performed a logistic regression analysis examining the association of complications with mortality at 30 postoperative days in 3 groups: (1) patients aged 65 years old and older linked to CMS data, (2) patients aged 65 years and older not linked to CMS data, and (3) patients aged younger than 65 years. We observed relatively consistent results across the 3 groups, particularly with respect to the 5 complications with the highest odds ratios (Table E1).

\section{DISCUSSION}

This study demonstrates that the adverse effects of operative complications on survival after lung cancer surgery predominantly manifest in the first 90 days after the operation and dissipate thereafter. Our analysis confirmed the presence of a differential magnitude of effect on survival for individual complications. In addition, time-varying effects on survival of individual complications after lung
TABLE 2. Frequencies of complications

\begin{tabular}{|c|c|c|}
\hline Complication & No. & $\% *$ \\
\hline Atrial arrhythmia & 4166 & 13.9 \\
\hline Blood transfusion & 2527 & 8.5 \\
\hline Pneumonia & 1285 & 4.3 \\
\hline Reintubation & 1134 & 3.8 \\
\hline Unexpected return to OR & 862 & 2.9 \\
\hline Delirium & 597 & 2.0 \\
\hline Acute kidney injury & 417 & 1.4 \\
\hline ARDS & 269 & 0.9 \\
\hline Ventricular arrhythmia & 248 & 0.8 \\
\hline Sepsis & 242 & 0.8 \\
\hline Initial ventilation $>48 \mathrm{~h}$ & 221 & 0.7 \\
\hline Central neurologic event & 212 & 0.7 \\
\hline Deep vein thrombosis & 176 & 0.6 \\
\hline Surgical site infection & 166 & 0.6 \\
\hline Pulmonary embolus & 145 & 0.5 \\
\hline Myocardial infarction & 139 & 0.5 \\
\hline Empyema & 126 & 0.4 \\
\hline Bronchopleural fistula & 112 & 0.4 \\
\hline Laryngeal nerve paralysis & 96 & 0.3 \\
\hline \multicolumn{3}{|l|}{ Mortality } \\
\hline $\begin{array}{l}\text { Operative }(30-\mathrm{d}+\text { in- } \\
\text { hospital })\end{array}$ & 728 & 2.4 \\
\hline 90-d & 1343 & 4.5 \\
\hline
\end{tabular}

OR, Operating room; ARDS, adult respiratory distress syndrome. *Percentage of all patients.

cancer surgery were demonstrated. Many complications, such as a myocardial infarction, were associated with an increased hazard of mortality in the immediate postoperative period but did not carry a significant hazard beyond 90 days after the operation. We believe that these estimated time-dependent hazard ratios can serve as objective weights for complications in future iterations of the STS GTSD risk and performance models for lung cancer surgery. Such an approach would represent an advance in quality measurement from the current assignment of equal weight to empirically selected major morbidities.

The 5-year survivals after surgical resection of a lung cancer for patients in the STS-CMS linked data set were as follows: stage I, $59.7 \%$; stage II, $40.7 \%$; stage III, $29.9 \%$; and stage IV, $26.7 \% .{ }^{4}$ We have previously demonstrated that individual patient and disease factors are associated with operative morbidity and mortality. ${ }^{2}$ Our group's recent work also shows that, as expected, long-term survival varies according to patient and disease factors as well. ${ }^{9}$ These data can help thoracic surgeons counsel patients regarding expected risks and outcomes with lung cancer 


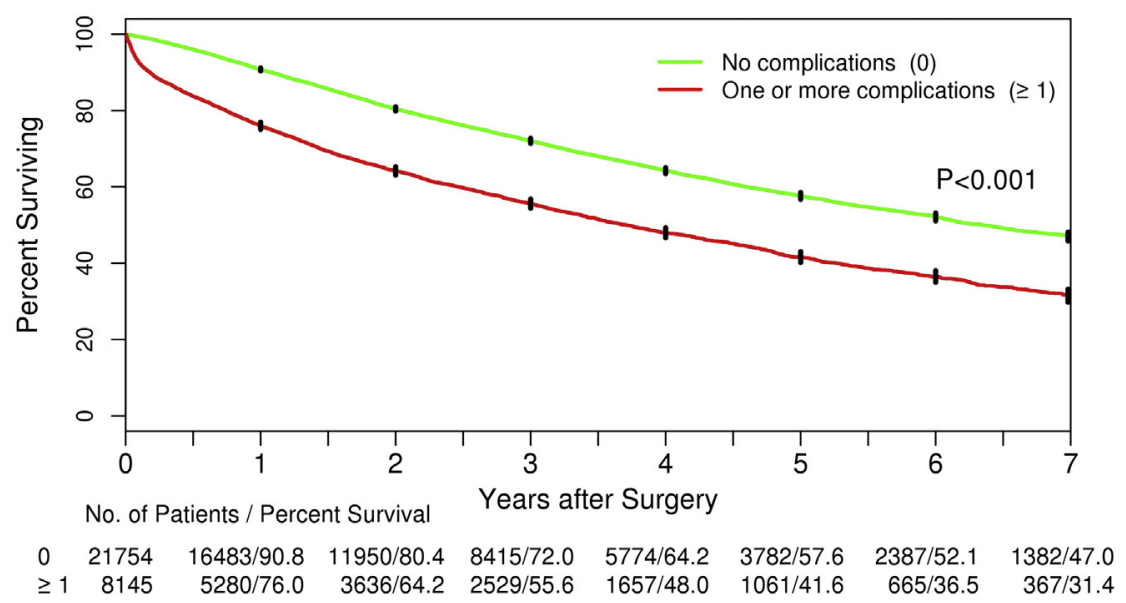

FIGURE 1. Unadjusted Kaplan-Meier survival curves for patients with and without one or more perioperative complications.

surgery. This study further refines these survival models by estimating the effects of the occurrence of postoperative complications on patient survival. This information can help direct quality improvement efforts toward prevention of the most harmful complications, and also enhance our ability to measure quality as discussed previously.

A
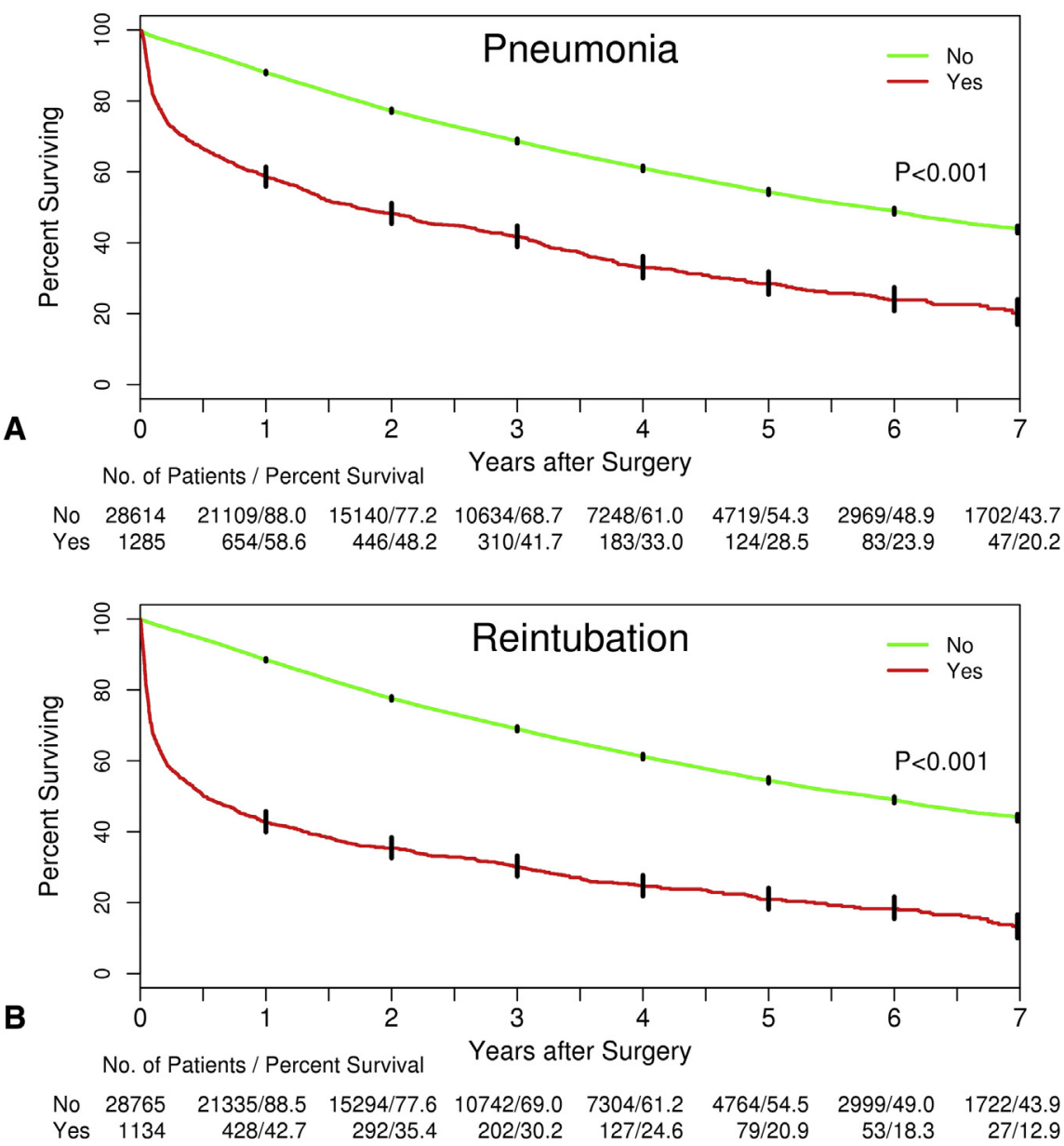

FIGURE 2. A, Unadjusted Kaplan-Meier survival curves for patients with and without pneumonia after lung cancer surgery. B, Unadjusted Kaplan-Meier survival curves for patients with and without reintubation after lung cancer surgery. 

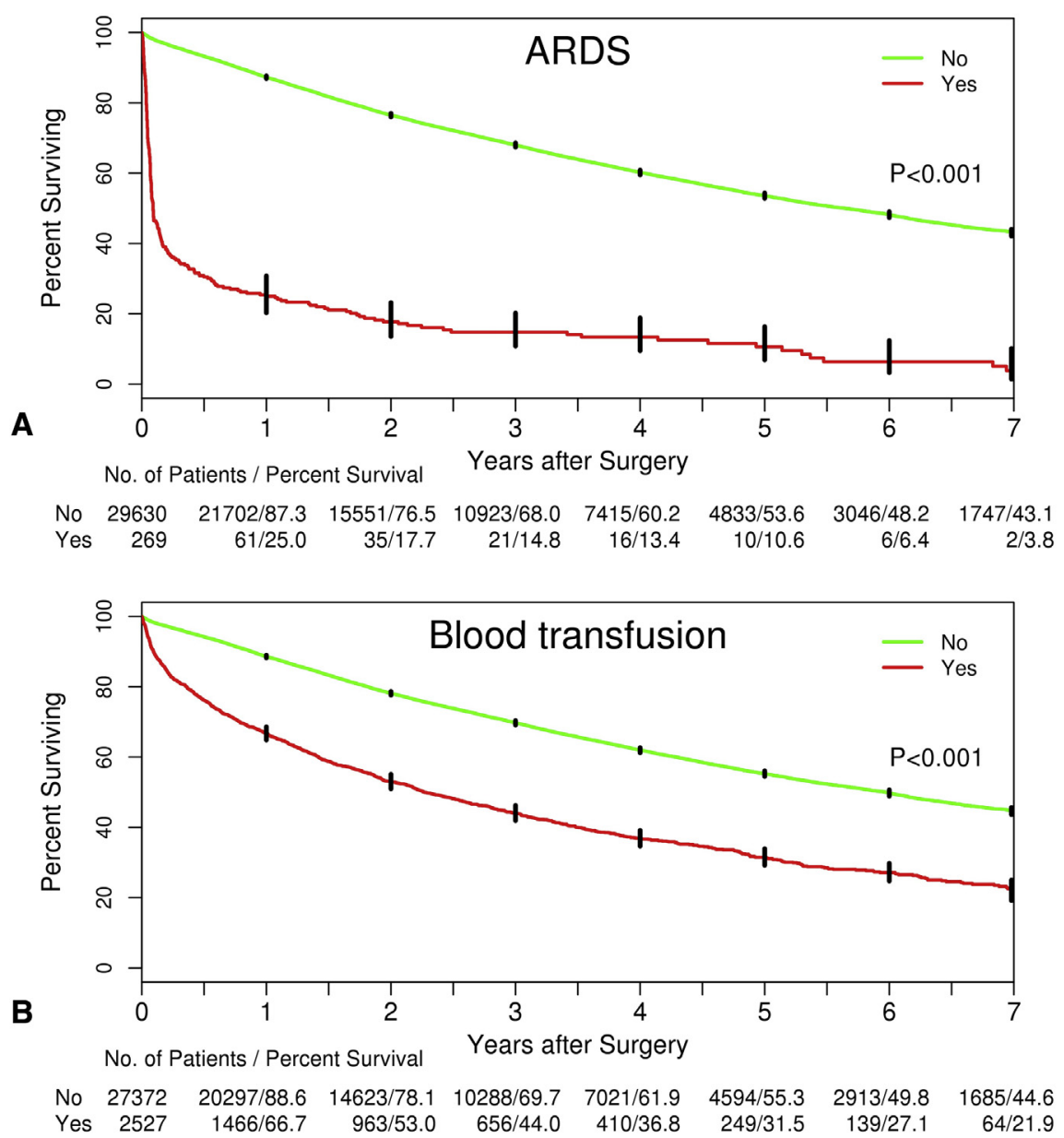

FIGURE 3. A, Unadjusted Kaplan-Meier survival curves for patients with and without adult respiratory distress syndrome (ARDS) after lung cancer surgery. B, Unadjusted Kaplan-Meier survival curves for patients with and without blood transfusion after lung cancer surgery.

The long-term effects of surgical complications after surgery for lung cancer have been previously examined by Rueth and colleagues ${ }^{1}$ in a study that used the Surveillance, Epidemiology and End Results-Medicare Database. In examining broad categories of complications in their analysis (any complication, pulmonary complication, cardiac complication, or noncardiopulmonary complication) derived from $I C D$-9-CM codes in the CMS record, they also found that complications independently affect longterm survival. In contrast to the analysis by Rueth and colleagues, ${ }^{1}$ our study analyzed the effects of specific individual complications with time (eg, pneumonia and ARDS are not grouped in a single category). Further, the STS GTSD provides much greater detail with respect to the operative complications, as well as the patient and disease variables that are adjusted for in the analysis. Finally, we considered the differential effects of complications on survival with time, whereas the analysis Rueth and colleagues $^{1}$ assumed a constant hazard of a specific complication (eg, atrial arrhythmia) with time. The association between postoperative complication and survival is not unique to lung cancer. In esophageal, colon, and pancreatic cancer, hospital readmission (a surrogate for postoperative complications), is also associated with worse long-term survival. ${ }^{10-12}$

We found that 12 different complications were associated with worse survival from 0 to 3 months after surgery. This decreases to 4 complications associated with worse survival in the interval from 3 to 18 months and only 2 complications (transfusion and sepsis) after 18 months. These data suggest that for many postoperative complications, once patients have been "rescued" from these complications, they may not carry a diminished long-term survival. This finding is important, because the variation in hospital mortality after lung cancer surgery has been shown to be more strongly related to rescuing patients from complications than to the occurrence of complications. ${ }^{13}$ For example, an acute pulmonary embolus may be associated with a significant hazard of mortality in the immediate perioperative period but have minimal impact on long term survival if survived. Both blood transfusions and sepsis after lung cancer surgery were associated with a 

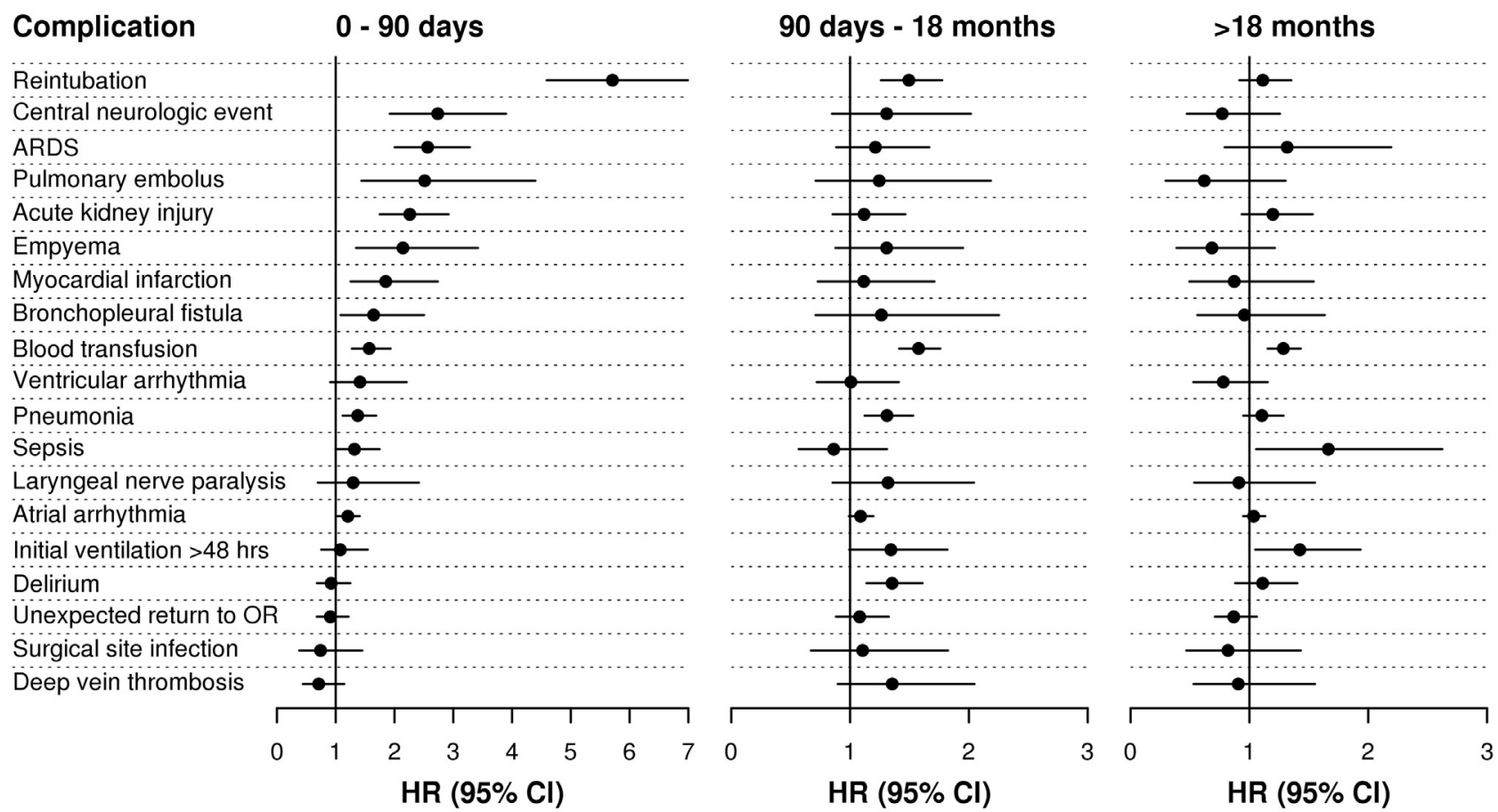

FIGURE 4. Differential magnitude of complications' impact on survival after lung cancer surgery shown with hazard ratios (HRs) varying with time. Numbers of patients at risk were 25,966 at the time of surgery (time 0), 23,910 at 90 days, and 15,903 at 18 months. ARDS, Adult respiratory distress syndrome; $O R$, operating room. $C I$, confidence interval.

worse long-term survival. One may hypothesize that these 2 complications exert an immunosuppressive effect that affects the risk of tumor progression. In fact, the association of postoperative blood transfusions with early tumor recurrence has been established for some time. ${ }^{14}$ Sepsis is associated with a significant hazard of mortality both in the early period of 0 to 90 days and the late period of longer than 18 months after surgery. The early effect may be the result of the physiologic stress of sepsis, whereas the late effect may be due to disease recurrence. Our data set does not contain information on cancer recurrence; the association between transfusions and increased risk of mortality after 18 months therefore cannot be investigated further. Contrary to our findings, a recent Surveillance, Epidemiology and End Results-Medicare analysis found that serious complications after complex cancer resections (pancreas, esophagus, and lung) are associated with a diminished long-term survival, even if the patient is rescued from the complication. ${ }^{15}$ That study differed from our analysis, however, in that complications were measured with claims data and a constant hazard of complications with time was assumed.

The STS National Database has adopted the use of composite measures of operative mortality plus major morbidity as outcome measures for risk models and performance measures developed for various operations., ${ }^{2,3,16-19}$ Analyzed operations in adult cardiac and thoracic surgery include lobectomy, esophagectomy, coronary artery bypass grafting, isolated valve surgery, and valve surgery plus coronary artery bypass grafting. The inclusion of major morbidities allows the measurement of many nonfatal events that are nevertheless very significant for patients. In addition, the inclusion of major morbidities in composite measures provides many more outcome events, which improves statistical modeling. The major morbidities used for these models are empirically determined by expert surgeon opinion. Complications considered minor are not included in the models. Further, equal weights are assigned to each major morbidity, and the presence of a major morbidity is considered an "all or none" event. Our approach will allow these major postoperative complications to be considered with specific weights in future risk models. The presence of multiple complications could potentially then be considered by summing the respective weights and examining interactions. We believe that this approach would represent an advance in our current method of risk assessment and performance measurement in cardiothoracic surgery. One question that remains uncertain is how best to weight complications relative to mortality. At present, in STS performance measures, mortality and major morbidity are weighted the inverse of their respective standard deviations. ${ }^{3}$

There are several important limitations to consider with respect to this study. First, the STS GTSD does not contain 
TABLE 3. Early ( $\leq 90$ days), middle ( $>90$ days-18 months), and late ( $>18$ months) hazard ratios for each postoperative complication after lung cancer resection

\begin{tabular}{|c|c|c|c|c|c|c|c|c|c|c|}
\hline \multirow[b]{2}{*}{ Complication } & \multicolumn{3}{|c|}{$0-90 \mathrm{~d}$} & \multicolumn{3}{|c|}{90 d-18 mo } & \multicolumn{3}{|c|}{$>18 \mathrm{mo}$} & \multirow[b]{2}{*}{ Overall $P$ value } \\
\hline & $\overline{H R}$ & $\mathbf{9 5} \% \mathrm{CI}$ & $P$ value & $\overline{\text { HR }}$ & $95 \% \mathrm{CI}$ & $P$ value & $\overline{\text { HR }}$ & $95 \% \mathrm{CI}$ & $P$ value & \\
\hline Reintubation & 5.71 & $4.59-7.10$ & $<.01$ & 1.50 & $1.26-1.78$ & $<.01$ & 1.11 & $0.92-1.35$ & .28 & $<.01$ \\
\hline Central neurologic event & 2.74 & $1.92-3.90$ & $<.01$ & 1.31 & $0.85-2.02$ & .22 & 0.77 & $0.47-1.26$ & .30 & $<.01$ \\
\hline ARDS & 2.56 & $2.00-3.28$ & $<.01$ & 1.21 & $0.88-1.67$ & .23 & 1.32 & $0.79-2.19$ & .29 & $<.01$ \\
\hline Pulmonary embolus & 2.51 & $1.44-4.40$ & .01 & 1.25 & $0.71-2.19$ & .44 & 0.62 & $0.29-1.30$ & .21 & $<.01$ \\
\hline Acute kidney injury & 2.26 & $1.75-2.92$ & $<.01$ & 1.12 & $0.85-1.47$ & .42 & 1.20 & $0.93-1.53$ & .15 & $<.01$ \\
\hline Empyema & 2.14 & $1.34-3.42$ & .01 & 1.31 & $0.88-1.95$ & .19 & 0.68 & $0.39-1.22$ & .20 & $<.01$ \\
\hline Myocardial infarction & 1.85 & $1.25-2.74$ & $<.01$ & 1.12 & $0.73-1.71$ & .62 & 0.87 & $0.49-1.54$ & .64 & .02 \\
\hline Bronchopleural fistula & 1.65 & $1.08-2.50$ & .02 & 1.26 & $0.71-2.25$ & .43 & 0.96 & $0.56-1.64$ & .87 & .12 \\
\hline Blood transfusion & 1.57 & $1.27-1.93$ & $<.01$ & 1.58 & $1.41-1.76$ & $<.01$ & 1.29 & $1.15-1.43$ & $<.01$ & $<.01$ \\
\hline Ventricular arrhythmia & 1.41 & $0.90-2.21$ & .13 & 1.01 & $0.72-1.41$ & .97 & 0.78 & $0.53-1.15$ & .21 & .18 \\
\hline Pneumonia & 1.37 & $1.12-1.69$ & $<.01$ & 1.31 & $1.12-1.53$ & $<.01$ & 1.10 & $0.95-1.29$ & .20 & $<.01$ \\
\hline Sepsis & 1.32 & $1.00-1.75$ & .05 & 0.86 & $0.57-1.31$ & .49 & 1.66 & $1.06-2.62$ & .03 & .03 \\
\hline Laryngeal nerve paralysis & 1.30 & $0.70-2.42$ & .41 & 1.32 & $0.85-2.04$ & .21 & 0.91 & $0.54-1.55$ & .73 & .47 \\
\hline Atrial arrhythmia & 1.21 & $1.03-1.41$ & .02 & 1.09 & $0.99-1.20$ & .08 & 1.04 & $0.95-1.13$ & .45 & .03 \\
\hline Initial ventilation $>48 \mathrm{~h}$ & 1.08 & $0.75-1.55$ & .68 & 1.34 & $0.99-1.82$ & .06 & 1.42 & $1.05-1.94$ & .02 & .05 \\
\hline Delirium & 0.92 & $0.68-1.25$ & .60 & 1.35 & $1.14-1.61$ & $<.01$ & 1.11 & $0.88-1.40$ & .38 & .01 \\
\hline Unexpected return to $\mathrm{OR}$ & 0.91 & $0.68-1.22$ & .52 & 1.08 & $0.88-1.33$ & .46 & 0.87 & $0.71-1.06$ & .17 & .35 \\
\hline Surgical site infection & 0.74 & $0.38-1.45$ & .38 & 1.11 & $0.67-1.82$ & .69 & 0.82 & $0.47-1.43$ & .49 & .71 \\
\hline Deep vein thrombosis & 0.71 & $0.44-1.15$ & .16 & 1.35 & $0.90-2.05$ & .15 & 0.91 & $0.53-1.55$ & .72 & .26 \\
\hline
\end{tabular}

$H R$, Hazard ratio; $C I$, confidence interval; $A R D S$, adult respiratory distress syndrome; $O R$, operating room.

data on the severity of complications. Not all pneumonias or myocardial infarctions, for example, would be expected to be of the same severity. The use of a grading system for the severity of surgical complications, such as the Clavien-Dindo classification, which has been validated in thoracic surgery, would further enhance this study as well as the STS GTSD. ${ }^{20,21}$ Second, we only consider the effects of complications on mortality. Complications can also significantly affect quality of life; however, such effects are not measured at present in the STS GTSD. Future incorporation of patient-reported outcomes into the STS GTSD will address this limitation. ${ }^{22}$ Further, although the effects of complications on survival are adjusted for individual patient characteristics and comorbidities, the potential exists for effect measure modification between clinical parameters and complications. For example, the effect of an episode of pneumonia may be more significant in a patient with a forced expiratory volume in 1 second that is $40 \%$ of predicted than a patient with a forced expiratory volume in 1 second that is $80 \%$ of predicted. These data are also limited to patients 65 years of age or older with Medicare insurance who received care at centers participating in the STS GTSD, which is thought to represent fewer than half of all patients undergoing lung cancer resection nationally. It is possible that the complications identified in this study may not have the same effect on survival in a younger patient population. As described in our first publication, $61 \%$ of patients in the STS GTSD for lung cancer surgery are aged 65 or older, and $70 \%$ of these are linked to CMS data. ${ }^{4}$ Therefore, $43 \%$ of patients in the STS GTSD for lung cancer surgery are included in the linked data set. Finally, lung cancer surgery outcomes reported in the GTSD have been shown to be superior to those reported from national administrative databases, and the findings may not be generalizable to patients undergoing lung cancer resection at institutions not participating in the STS GTSD. ${ }^{23}$

In conclusion, after lung cancer surgery, operative complications have a differential effect on long-term survival dependent on the specific complication. Further, the effect of a specific complication varies with time, with the hazard decreasing with time for certain complications (eg, pneumonia) and increasing for others (eg, sepsis). We believe that the use of these estimated time-dependent hazard ratios as objective weights for postoperative complications may improve risk assessment and performance measurement models in cardiothoracic surgery. The identification of the complications with the greatest effects (most harmful) can also help guide quality improvement initiatives in lung cancer surgery. 


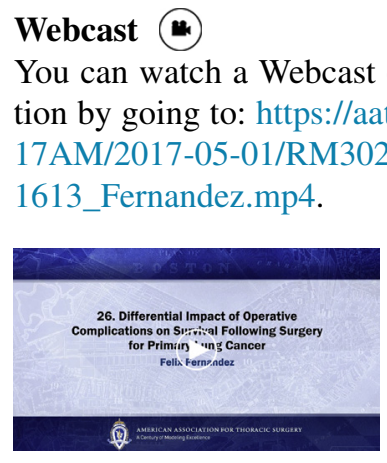

\section{Conflict of Interest Statement}

Authors have nothing to disclose with regard to commercial support.

\section{References}

1. Rueth NM, Parsons HM, Haberman EB, Groth SS, Virnig BA, Tuttle TM, et al. The long-term impact of surgical complications after resection of stage I nonsmall cell lung cancer: a population-based survival analysis. Ann Surg. 2011; 254:368-74.

2. Fernandez FG, Kosinski AS, Burfeind W, Park B, DeCamp MM, Seder C, et al. The Society of Thoracic Surgeons lung cancer resection risk model: higher quality data and superior outcomes. Ann Thorac Surg. 2016;102:370-7.

3. Kozower BD, O’Brien SM, Kosinski AS, Magee MJ, Dokholyan R, Jacobs JP, et al. The Society of Thoracic Surgeons composite score for rating program performance for lobectomy for lung cancer. Ann Thorac Surg. 2016;101:1379-86; discussion 1386-7.

4. Fernandez FG, Furnary AP, Kosinski AS, Onaitis MW, Kim S, Boffa D, et al. Longitudinal follow-up of lung cancer resection from the Society of Thoracic Surgeons General Thoracic Surgery Database in patients 65 years and older. Ann Thorac Surg. 2016;10 1:2067-76.

5. Magee MJ, Wright CD, McDonald D, Fernandez FG, Kozower BD. External validation of the society of thoracic surgeons general thoracic surgery database. Ann Thorac Surg. 2013;96:1734-9; discussion 1738-9.

6. Therneau TM, Grambsch PM. Modeling Survival Data: Extending The Cox Model. New York: Springer-Verlag; 2000.

7. Lee EW, Wei LJ, Amato DA. Cox-type regression analysis for large numbers of small groups of correlated failure time observations. In: Klein JP, Goel PK, eds. Survival analysis: state of the art. Dordrecht, Netherlands: Kluwer Academic Publishers; 1992:237-47.

8. R Core Team. R: A Language and Environment For Statistical Computing. Vienna, Austria: R Foundation for Statistical Computing; 2014. Available at: http://www.R-project.org/. Accessed February 2, 2017.

9. Onaitis MW, Furnary AP, Kosinski AS, Kim S, Boffa D, Cowper P, et al. Prediction of long-term survival following lung cancer surgery for elderly patients in the Society of Thoracic Surgeons General Thoracic Surgery Database. Ann Thor Surg. 2018;105:309-16.

10. Fernandez FG, Khullar O, Force SD, Jiang R, Pickens A, Howard D, et al. Hospital readmission is associated with poor survival after esophagectomy for esophageal cancer. Ann Thorac Surg. 2015;99:292-7.

11. Tevis SE, Kohlnhofer BM, Stringfield S, Foley EF, Harms BA, Heise CP, et al. Postoperative complications in patients with rectal cancer are associated with delays in chemotherapy that lead to worse disease-free and overall survival. Dis Colon Rectum. 2013;56:1339-48.

12. Stitzenberg KB, Chang Y, Smith AB, Nielsen ME. Exploring the burden of inpatient readmissions after major cancer surgery. J Clin Oncol. 2015;33:455-64.

13. Farjah F, Backhus L, Cheng A, Englum B, Kim S, Saha-Chaudhuri P, et al. Failure to rescue and pulmonary resection for lung cancer. J Thorac Cardiovasc Surg. 2015; 149:1365-71; discussion 1371-3.e3.

14. Blumberg N, Heal J, Chuang C, Murphy P, Agarwal M. Further evidence supporting a cause and effect relationship between blood transfusion and earlier cancer recurrence. Ann Surg. 1988;207:410-5.

15. Nathan H, Yin H, Wong SL. Postoperative complications and long-term survival after complex cancer resection. Ann Surg Oncol. 2017;24:638-44.
16. Shahian DM, O'Brien SM, Filardo G, Ferraris VA, Haan CK, Rich JB, et al; Society of Thoracic Surgeons Quality Measurement Task Force. The Society of Thoracic Surgeons 2008 cardiac surgery risk models: part 1—coronary artery bypass grafting surgery. Ann Thorac Surg. 2009;88(1 Suppl):S2-22.

17. O'Brien SM, Shahian DM, Filardo G, Ferraris VA, Haan CK, Rich JB, et al; Society of Thoracic Surgeons Quality Measurement Task Force. The Society of Thoracic Surgeons 2008 cardiac surgery risk models: part 2-isolated valve surgery. Ann Thorac Surg. 2009;88(1 Suppl):S23-42.

18. Shahian DM, O'Brien SM, Filardo G, Ferraris VA, Haan CK, Rich JB, et al; Society of Thoracic Surgeons Quality Measurement Task Force. The Society of Thoracic Surgeons 2008 cardiac surgery risk models: part 3-valve plus coronary artery bypass grafting surgery. Ann Thorac Surg. 2009;88(1 Suppl): S43-62.

19. Raymond DP, Seder CW, Wright CD, Magee MJ, Kosinski AS, Cassivi SD, et al. Predictors of major morbidity or mortality after resection for esophageal cancer: a Society of Thoracic Surgeons General Thoracic Surgery Database risk adjustment model. Ann Thorac Surg. 2016;102:207-14.

20. Clavien PA, Barkun J, de Olivera ML, Vauthey JN, Dindo D, Schulick RD, et al. The Clavien-Dindo classification of surgical complications: five-year experience. Ann Surg. 2009;250:187-96.

21. Seely AJ, Ivanovic J, Threader J, Al-Hussaini A, Al-Shehab D, Ramsay T, et al. Systematic classification of morbidity and mortality after thoracic surgery. Ann Thorac Surg. 2010;90:936-42; discussion 942.

22. Khullar OV, Rajaei MH, Force SD, Binongo JN, Lasanajak Y, Robertson S, et al. Pilot study to integrate patient reported outcomes after lung cancer surgery into the STS database. Ann Thor Surg. 2017;104:245-53.

23. LaPar DJ, Bhamidipati CM, Lau CL, Jones DR, Kozower BD. The Society of Thoracic Surgeons General Thoracic Surgery Database: establishing generalizability to national lung cancer resection outcomes. Ann Thorac Surg. 2012;94: 216-21; discussion 221.

Key Words: lung cancer surgery

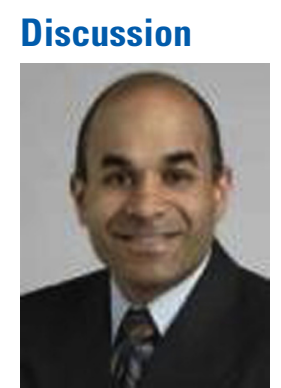

Dr Sudish Murthy (Cleveland, Ohio). Thanks, Dr Fernandez, for a very nice article. I appreciate getting the manuscript ahead of time, and I thank the Association for the invitation.

There is little doubt that these big database studies are becoming increasingly prevalent in our meetings, as we have heard today, and also in the journals that we read. There are numerous vagaries that come along with the use of these database studies, however, and it is largely because there is some problem in granularity or some problem in long-term follow-up or outcome. Fernandez and colleagues have attempted to make up or mitigate some of the deficiencies of the STS database by merging it with CMS data. This is not the first time that they have used this methodology; they have validated it before.

Specifically, Fernandez and colleagues are using this approach in an attempt to hierarchically rank postoperative complications that occur after lung resection for cancer to determine the impact on survival of these unique complications and put some real teeth into quality metrics, which are currently assigning equal weights to many of these complications. They are also adding a longitudinal component to their analytics to gain insight into some of the late hazards. 
Dr Fernandez, I have some questions for you. There are a few vagaries even in the STS database. How has the evolution of the STS database through its various versions affected your analysis?

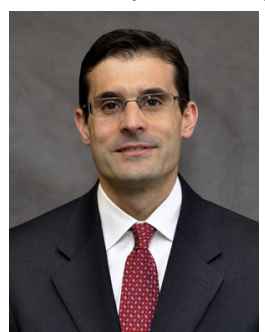

Dr Fernandez. At this point, it has affected it very little. We did spend a lot of time before we commenced any of these studies reconciling various versions of the database. If you look at our first publication that came from this work, it really goes into detail on how we analyzed this and reconciled different data elements into a seamless data set that can be analyzed. But that was a very important consideration.

Dr Murthy. How much of the data do you think that you lose when you merge the databases, roughly, and how do you think that the outcomes for those patients who were not Medicare patients could potentially warp the final conclusions of a study like this?

Dr Fernandez. That's another very good question. These data represent $41 \%$ to $42 \%$ of the entire STS lung cancer surgery cohort. The median age is 68 years, if you take it altogether, but it is 65 years old or greater. When we link the data, we lose about $30 \%$ of patients who were covered by private insurance or health maintenance organizations. So that ends up being $42 \%$ of $43 \%$ of the lung cancer surgery population that we can analyze. In the 65-year-old and older crowd, the two groups are similar in terms of demographics, stage, treatment, and short-term outcomes; however, if you compare them with the younger cohort, they have lower operative mortality, they have a tendency toward higher stage tumors, and they tend to undergo more aggressive resections, such as pneumonectomies and sleeves.

Dr Murthy. The magnitude of the hazards for late mortality seems to be significantly lower than that of the hazards for the early ones. Does this call into question the direct effects of these complications on late mortality and make you a little less confident of any true cause-and-effect scenario for the later hazards?

Dr Fernandez. Let me answer your question as I understand it. A lot of these complications really have an effect early on, whether it is the severity of the complication and maybe how it interacts with patient comorbidity, which places a hazard on mortality early on or may be a characteristic of the centers and the concept of failure to rescue; probably a combination of both. But it seems that if these complications are survived in the short term, their hazard significance dissipates with time.

You mentioned the late term, and sepsis and blood transfusion are the only factors that are significant at that time. If you look at the unadjusted survival curves, they become parallel after some point, so this makes sense. The sepsis and blood transfusion need more study, but one could think of immunologic mechanisms driving tumor recurrence, and certainly blood transfusions have been associated with cancer recurrence in previous studies. Unfortunately, our data set does not allow us to study disease-free recurrence or cancer-specific survival.

Dr Murthy. How do you account for patients who have died of their complications early possibly being censored from later analysis, and how do you make up for this competing risk of death that may somehow reduce the impact of these complications if they are survived early to affect late survival?

Dr Fernandez. That's a good question, too. With this time-dependent coefficient, because the proportional hazards assumption is violated, each period starts a new model, essentially, with the population at risk. How we account for competing causes of death is by adjustment in the model for the relevant tumor stage, age, and comorbidity, treatment variables that are included in the STS database. Thirtysome variables that are in there and adjusted for, and as well as those variables adjust, that should account for balance and competing causes of death. So, we are subject to unmeasured confounding, such as any other retrospective analysis of this nature, but that is how that is adjusted for.

Dr Murthy. Predicting outcomes from outcomes is always a tricky business. When you are a patient and you see some of these data, you are not sure whether you are going to get the blood transfusion or get the pneumonia. How do we use this new information from a prognostic or from an information standpoint that we would then give our patients?

Dr Fernandez. I think the best way we use this is to help us measure performance better. Our short-term lung cancer surgery models are better than administrative data, but they need improvement. We cannot use mortality as the only outcome measure, because it is rare. So these composite measures have been created that include some complications that are selected by expert opinion that increase the outcomes to allow for power for statistical modeling. We need to determine how to use these better, how to weight them better, so that when you get your star rating, this will hopefully, if implemented, improve discrimination. There is much more improvement that needs to be done. We need to grade complications, and we need to start considering outcome measures that extend beyond the short-term outcomes of morbidity and mortality, but it is an iterative process of continuing to improve what we have.

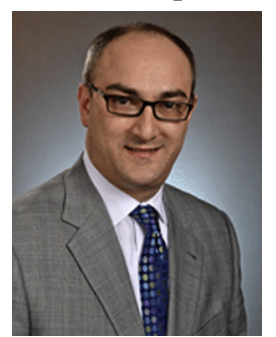

Dr Michael Ebright (New York, NY). Dr Fernandez, thank you very much. It was a great presentation. I also think it is very timely.

I want to get back to the concept of causality, about how we can use this information prospectively with our patients. I am wondering whether your database work had the resolution to determine, for instance, 
whether delirium was simply a surrogate for advanced age, whether reintubation was simply a surrogate for poor selection of patients with poor PFPs and whether you can link that.

Dr Fernandez. I agree with you. We used the variables that have been collected in the STS database for a period of greater than a decade. So reintubation is certainly a surrogate for respiratory failure and probably relates back to patient selection, and delirium is probably confounded by several other factors as well. We are only able to use the data that we have available to us. But I would agree with you.

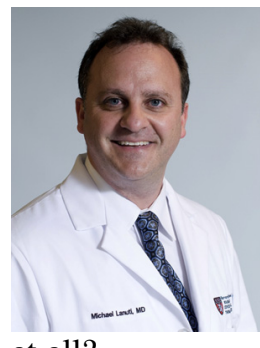

Dr Michael Lanuti (Boston, Mass). Dr Fernandez, congratulations on the article, and it will be another contribution for this body of work that you are creating. I noticed that you did not put in prolonged postoperative air leak. I know that the STS captures it, but did that drop out or was it analyzed at all?

Dr Fernandez. That was not included in the model.

Dr Lanuti. Although it is something that the general thoracic database captures, correct?
Dr Fernandez. That is a variable in the database, yes.

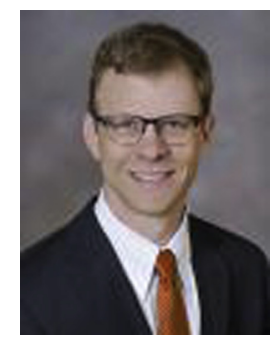

Dr Paul H. Schipper (Portland, Ore). My question is an expansion of that. You chose complications. The STS database tracks a lot more than what you chose. In particular, something like chylothorax may have that same late-term mortality that you see with transfusion and ARDS, and I am wondering whether you can talk a little bit more about how you chose the complications to put into your study.

Dr Fernandez. I guess I would just say that the variables that we selected for inclusion into this model were selected by consensus opinion of our research group, and we selected 19 variables. Certainly, we could add other ones in there. You look at the frequency of these; pneumonia is only 4\%, acute kidney injury is only $1.4 \%$. We do lung cancer with low mortality and low morbidity. It is safe; there are not a lot of events. Given our sample size, we have a lot of statistical power, so most things you enter into the model are probably going to show some significance. 
TABLE E1. Logistic regression analysis examining the association of complications with mortality at the 30 postoperative days in patients age 65 years old and older linked to Centers for Medicare and Medicaid Services data, in patients age 65 years old and older not linked to Centers for Medicare and Medicaid Services data, and in all patients older than 65 years

\begin{tabular}{|c|c|c|c|c|c|c|c|c|c|}
\hline \multirow[b]{2}{*}{ Complication } & \multicolumn{3}{|c|}{ Age $\geq 65$ years linked to CMS } & \multicolumn{3}{|c|}{ Age $\geq 65$ years not linked to CMS } & \multicolumn{3}{|c|}{ Age $<65$ years } \\
\hline & OR & $95 \% \mathrm{CI}$ & $P$ value & OR & $95 \% \mathrm{CI}$ & $P$ value & $\overline{\mathrm{OR}}$ & $95 \% \mathrm{CI}$ & $P$ value \\
\hline Reintubation & 9.94 & $7.13-13.84$ & $<.01$ & 12.94 & $7.78-21.51$ & $<.01$ & 7.73 & $4.74-12.61$ & $<.01$ \\
\hline Central neurologic event & 6.52 & $3.83-11.11$ & $<.01$ & 6.46 & $2.45-17.07$ & $<.01$ & 4.45 & $1.57-12.65$ & .01 \\
\hline ARDS & 4.96 & $3.23-7.63$ & $<.01$ & 4.68 & $2.84-7.70$ & $<.01$ & 6.36 & $3.29-12.27$ & $<.01$ \\
\hline Pulmonary embolus & 5.38 & $2.72-10.65$ & $<.01$ & 1.56 & $0.44-5.58$ & .49 & 4.04 & $1.48-11.02$ & .01 \\
\hline Acute kidney injury & 4.35 & $3.05-6.20$ & $<.01$ & 2.98 & $1.87-4.73$ & $<.01$ & 2.33 & $1.06-5.14$ & .04 \\
\hline Empyema & 2.39 & $1.15-4.98$ & .02 & 2.44 & $0.90-6.57$ & .08 & 0.46 & $0.08-2.65$ & .38 \\
\hline Myocardial infarction & 2.97 & $1.25-7.09$ & .01 & 2.65 & $1.03-6.77$ & .04 & 2.86 & $0.63-13.01$ & .17 \\
\hline Bronchopleural fistula & 1.93 & $0.87-4.30$ & .11 & 0.75 & $0.30-1.90$ & .54 & 2.01 & $0.75-5.39$ & .16 \\
\hline Blood transfusion & 1.42 & $1.06-1.89$ & .02 & 1.61 & $1.11-2.34$ & .01 & 1.81 & $1.18-2.79$ & .01 \\
\hline Ventricular arrhythmia & 2.70 & $1.57-4.65$ & $<.01$ & 5.35 & $2.50-11.49$ & $<.01$ & 1.76 & $0.56-5.57$ & .33 \\
\hline Pneumonia & 1.40 & $1.00-1.97$ & .05 & 0.79 & $0.47-1.31$ & .36 & 0.87 & $0.51-1.49$ & .62 \\
\hline Sepsis & 2.66 & $1.66-4.28$ & $<.01$ & 2.70 & $1.45-5.03$ & $<.01$ & 5.85 & $2.95-11.61$ & $<.01$ \\
\hline Laryngeal nerve paralysis & 0.29 & $0.07-1.24$ & .09 & 0.85 & $0.12-6.21$ & .87 & 0.28 & $0.05-1.54$ & .14 \\
\hline Atrial arrhythmia & 1.09 & $0.86-1.37$ & .49 & 1.06 & $0.73-1.54$ & .75 & 1.19 & $0.77-1.82$ & .43 \\
\hline Initial ventilation $>48 \mathrm{~h}$ & 1.18 & $0.57-2.44$ & .66 & 1.93 & $1.00-3.73$ & .05 & 3.01 & $1.29-6.99$ & .01 \\
\hline Delirium & 0.79 & $0.49-1.29$ & .35 & 0.99 & $0.45-2.18$ & .97 & 0.66 & $0.28-1.55$ & .34 \\
\hline $\begin{array}{l}\text { Unexpected return to } \\
\text { operating room }\end{array}$ & 1.19 & $0.78-1.83$ & .42 & 2.39 & $1.42-4.05$ & .01 & 1.49 & $0.78-2.83$ & .22 \\
\hline Surgical site infection & 0.38 & $0.09-1.52$ & .17 & 0.49 & $0.07-3.60$ & .49 & 1.03 & $0.16-6.64$ & .98 \\
\hline Deep vein thrombosis & 1.12 & $0.47-2.65$ & .80 & 0.55 & $0.17-1.75$ & .31 & 0.73 & $0.29-1.88$ & .52 \\
\hline
\end{tabular}

$C M S$, Centers for Medicare and Medicaid Services data; $O R$, odds ratio; $C I$, confidence interval; $A R D S$ adult respiratory distress syndrome. 